\title{
Heartbeat: interaction of renin-angiotensin-aldosterone blocking drugs with COVID-19 disease susceptibility and severity
}

Angiotensin converting enzyme 2 (ACE2) is the primary receptor for the SARS-CoV-2 virus raising concern that patients with cardiovascular disease being treated with an ACE inhibitor or angiotensin receptor blocker (ARB) drug might have altered ACE2 expression leading to increased risk of COVID-19 infection or a more severe disease course. In this issue of Heart, Hippisley-Cox and colleagues ${ }^{1}$ addressed this question using routinely collected data in a prospective cohort study in England of 8.28 million participants aged 20-99 years. COVID-19 disease was diagnosed in 19486 patients and 1286 required treatment in the intensive care unit (ICU). Even after adjustment for confounders, ACE inhibitors were associated with a significantly reduced risk of COVID-19 disease (adjusted HR 0.71, 95\% CI 0.67 to 0.74 ) and no increased risk of ICU care (adjusted HR 0.89, 95\% CI 0.75 to 1.06). Similarly, adjusted HRs for ARBs were 0.63 (95\% CI 0.59 to 0.67 ) for COVID-19 disease and 1.02 (95\% CI 0.83 to 1.25) for ICU care. However, there were significant interactions with ethnicity for ACE inhibitors and ARB (both $\mathrm{p}<0.001$ ) for COVID-19 RT-PCR diagnosed disease outcome (table 1).

In the accompanying editorial, Straw and Witte $^{2}$ point out that 'Observational data are challenging to work with, requiring care to avoid the pitfalls of observed and hidden interactions and biases while balancing the need to make the results accessible to nonstatisticians. The reliability of conclusions drawn from observational data depends on their interpretation and on the data themselves.' However, given the rigorous approach in this study, they that this data 'should reassure physicians and patients that these vital medications are not implicated in COVID-19 and should not be stopped.' This study also underscores the principles 'that the cardiovascular system is not the same across ethnicities, that good supportive care in the absence of specific therapies is essential and that the independent scientific process based on precise peer review must be protected, even when authors and editors are under immense pressure.'

Correspondence to Professor Catherine M Otto, Division of Cardiology, University of Washington, Seattle, WA 98195, USA; cmotto@uw.edu

Table 1 Adjusted HRs $(95 \% \mathrm{Cl})$ for risk of COVID-19 positive test associated with ACE inhibitor and ARB exposure by ethnic group

\begin{tabular}{llllll}
\hline & \multicolumn{2}{l}{ ACE inhibitor } & & ARB & \\
\cline { 2 - 2 } & Adjusted HR $(95 \% \mathrm{Cl})$ & P value & & Adjusted HR (95\% Cl) & P value \\
\hline White & $0.66(0.63$ to 0.70$)$ & $<0.001$ & $0.56(0.52$ to 0.62$)$ & $<0.001$ \\
Indian & $0.74(0.61$ to 0.90$)$ & 0.003 & $0.66(0.52$ to 0.82$)$ & $<0.001$ \\
\hline Pakistani & $0.83(0.64$ to 1.09$)$ & 0.182 & $0.78(0.57$ to 1.06$)$ & 0.114 \\
\hline Bangladeshi & $0.97(0.72$ to 1.31$)$ & 0.847 & $0.74(0.49$ to 1.13$)$ & 0.164 \\
\hline Other Asian & $0.81(0.64$ to 1.03$)$ & 0.084 & $0.96(0.73$ to 1.23$)$ & 0.726 \\
Caribbean & $1.05(0.87$ to 1.28$)$ & 0.480 & $0.70(0.53$ to 0.92$)$ & 0.010 \\
Black African & $1.31(1.08$ to 1.59$)$ & 0.005 & $1.24(0.99$ to 1.58$)$ & 0.062 \\
\hline Chinese & $0.73(0.30$ to 1.79$)$ & 0.575 & $1.53(0.77$ to 3.01$)$ & 0.223 \\
\hline Other ethnic group & $0.82(0.67$ to 1.05$)$ & 0.122 & $1.09(0.86$ to 1.39$)$ & 0.475 \\
\hline
\end{tabular}

HRs are comparing risks of COVID-19 in users versus non-users of ACE inhibitor and ARB, and are adjusted for age, sex, deprivation, geographical region, comorbidities (including hypertension included as a binary variable) and other medications listed in table 1 of Hippisley-Cox and colleagues. ${ }^{1}$

$A R B$, angiotensin receptor blocker.

Similarly, a meta-analysis of 10 studies enrolling 9890 hypertensive subjects found that the risk of severe or lethal COVID-19 was no increased in patients on treatment with an ACE inhibitor or ARB compared with patients not on these medications (for
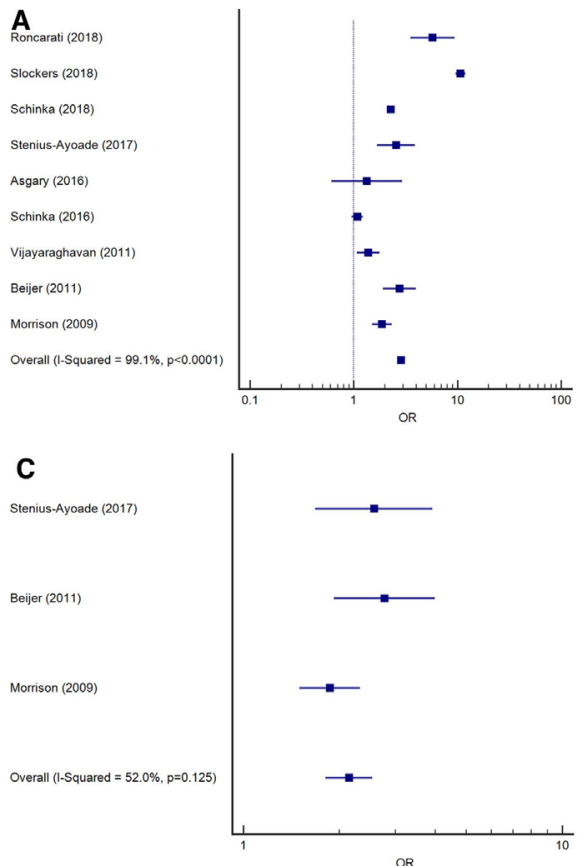

Figure 1 Forest plots of ORs of cardiovascular disease (CVD) (International Classification of Diseases, Tenth Revision definition) in homeless versus housed individuals from (A) all studies in meta-analysis ( $n=9)$, (B) North American studies $(n=6)$ and (C) European studies $(n=3)$. (D) Forest plot of ORs of hypertension (a subset of CVD) ( $n=2)$.

ACE inhibitors OR: $0.90 ; 95 \% \mathrm{CI} 0.65$ to 1.26; for ARBs OR 0.92 ; $95 \%$ CI 0.75 to $1.12)^{3}$

These two studies in Heart complement the randomised clinical trial (RCT) recently presented at the European Society
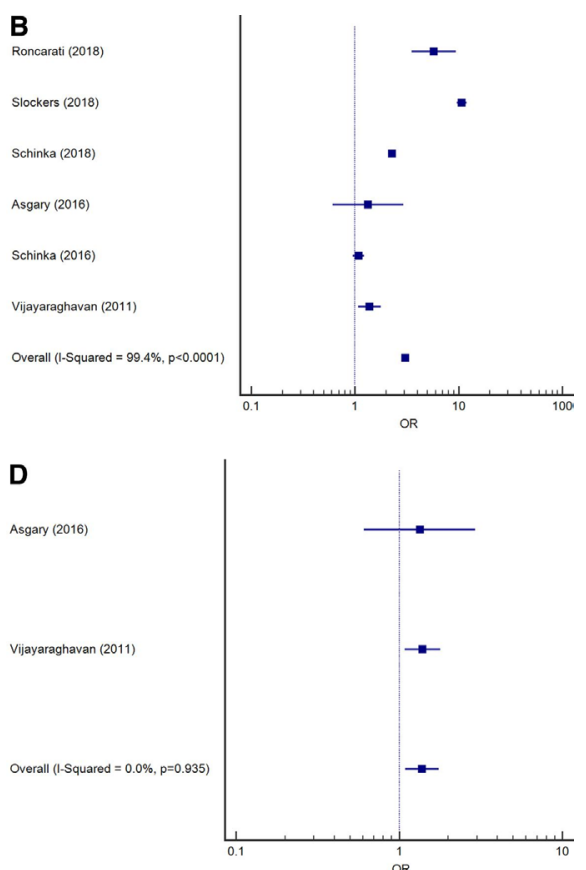
of Cardiology Annual Scientific Sessions. In the Angiotensin Receptor Blockers and Angiotensin-converting Enzyme Inhibitors and Adverse Outcomes in Patients With COVID19 (BRACE-CORONA) trial, ${ }^{4}$ 659 patients hospitalized with COVID-19 infection who were chronically on an ACE inhibitor or ARB were randomised to 30-days of suspension vs continuation of these medications. The mean age was relatively young (56 years) and 40\% were women, with hypertension in 100\% but heart failure in only $1.4 \%$. Overall, thehe primary outcomeof days alive and out of the hospital at 30 days was no different in those suspending or contining ACEinhibitor or ARB therapy. The congruence between this RCT and the publications in this issue of Heart shows the power of carefully performed and analyzed oberservational studies.

Homelessness is an oftenunderappreciated risk factor for cardiovascular disease (CVD). 'Homelessness is a global crisis which has increased at an alarming rate over the last decade; worldwide it is estimated that nearly 1.6 billion people have inadequate shelter, resulting in the United Nations announcing its firstever resolution on homelessness on 19 February 2020. ${ }^{5}$ In a meta-analysis of 17 articles addressing both the risk of CVD and interventions for CVD in homeless

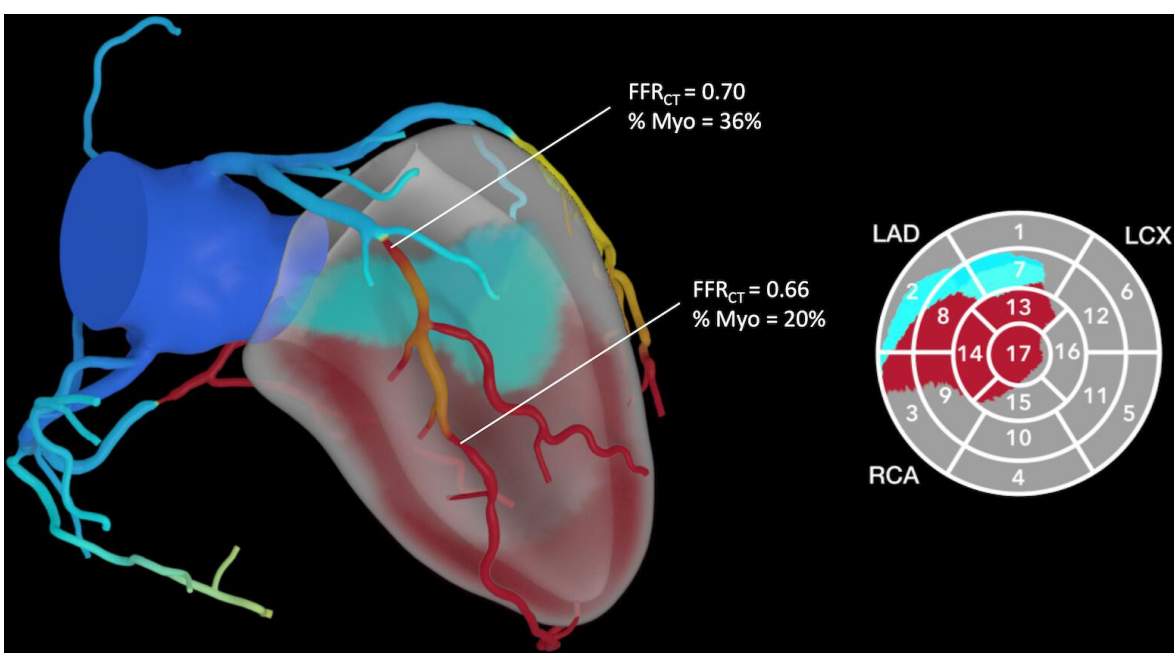

Figure 2 Integrating fractional myocardial mass with $\mathrm{FFR}_{\mathrm{CT}}$ analysis. A conceptual image of how integrating $\mathrm{FFR}_{\mathrm{CT}}$ with myocardium at risk and myocardial blood flow could be applied in future iterations of CTCA analysis. CTCA, CT coronary angiography; FFR, fractional flow reserve; $F F R_{\mathrm{CT}^{\prime}} \mathrm{CT}$ derived FFR; LAD, left anterior descending.

populations, Al-Shakarchi and colleagues ${ }^{6}$ found an almost threefold increased risk of CVD in homeless individuals pooled (OR 2.96 ; $95 \%$ CI 2.80 to 3.13 ; p $<0.0001$ ) and a higher CVD mortality rate than nonhomeless individuals(age-standardised mortality ratio range: 2.6-6.4) (figure 1).

As Lane concludes in her editorial, 'We need to gain a better understanding of the magnitude and types of CVD among the

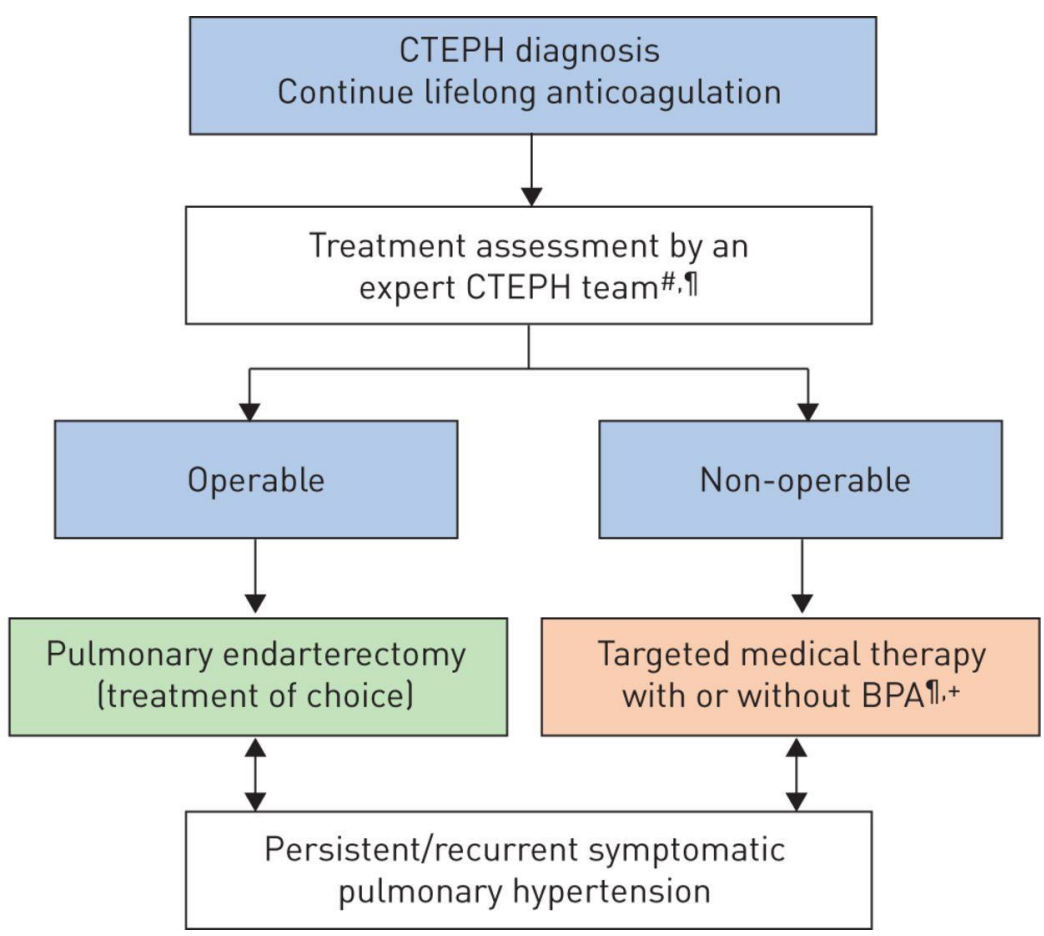

Figure 3 Chronic thromboembolic pulmonary hypertension (CTEPH) treatment algorithm. ${ }^{10}$ \#Multidisciplinary: pulmonary endarterectomy surgeon, pulmonary hypertension (PH) expert, BPA interventionist and radiologist. ๆTreatment assessment may differ depending on the level of expertise. +BPA without medical therapy can be considered in selected cases. BPA, balloon pulmonary angioplasty. homeless; the specific barriers to healthcare faced by homeless people; and identify effective models of care and adapt/tailor these into current CVD services and existing homeless services.' 'One important tangible difference healthcare professionals can implement immediately is to treat everyone with the same level of professionalism and compassion and not discriminate or stigmatise people who are homeless.'

An interesting imaging study by Keulards and colleagues ${ }^{7}$ demonstrates that CT flow-based measurement of myocardial mass is closely related to invasively measured myocardial perfusion supporting the use of CT-mass for estimating the extent of myocardium-at-risk in patients with proximal coronary artery disease. The novelty of this approach is put into context by Ihdayhid and Sellers ${ }^{8}$ who discuss how fractional flow reserve measured by CT $\left(\mathrm{FFR}_{\mathrm{CT}}\right)$ techniques might allow us to 'integrate both physiology and myocardium at risk into a single noninvasive test' (figure 2).

The Education in Heart article in this issue ${ }^{9}$ provides an overview of interventional approaches to treatment of chronic thromboembolic pulmonary hypertension (CTEPH), comparing surgical pulmonary endarterectomy (PEA) versus balloon pulmonary angioplasty (BPA) (figure 3). As they point out 'BPA is now sufficiently developed that appropriately powered RCTs comparing PEA versus BPA for segmental and subsegmental (levels 3 and 4) CTEPH could be planned in centres with equivalent experience in both techniques. However, consensus in the interpretation of imaging and standardisation of the surgical and BPA approach between 
centres will be important to deliver meaningful comparisons.'

Funding The authors have not declared a specific grant for this research from any funding agency in the public, commercial or not-for-profit sectors.

Competing interests None declared.

Patient and public involvement Patients and/or the public were not involved in the design, or conduct, or reporting, or dissemination plans of this research.

Patient consent for publication Not required.

Provenance and peer review Commissioned; internally peer reviewed.

(C) Author(s) (or their employer(s)) 2020. No commercial re-use. See rights and permissions. Published by BMJ.

A) Check for updates

To cite Otto CM. Heart 2020;106:1451-1453.
Heart 2020;106:1451-1453.

doi:10.1136/heartjnl-2020-318164

\section{ORCID iD}

Catherine M Otto http://orcid.org/0000-0002-05279392

\section{REFERENCES}

1 Hippisley-Cox J, Young D, Coupland C, et al. Risk of severe COVID-19 disease with ACE inhibitors and angiotensin receptor blockers: cohort study including 8.3 million people. Heart 2020;106:1503-11.

2 Straw S, Witte KK. Observational data during the COVID-19 pandemic: opportunity with uncertainty. Heart 2020;106:1461-2.

3 Flacco ME, Acuti Martellucci C, Bravi F, et al. Treatment with ACE inhibitors or Arbs and risk of severe/lethal COVID-19: a meta-analysis. Heart 2020;106:1519-24.

4 Lopes RD on behalf of the BRACE CORONA Investigators. Continuing versus suspending $\mathrm{ACE}$ inhibitors and ARBs: Impact of adverse outcomes in hospitalized patients with COVID-19-The BRACE CORONA Trial. Presented at ESC Scientific Sessions: Sept 1, 2020.

5 Lane DA. Homelessness: a 'cause of the causes' of cardiovascular disease? Heart 2020:106:1456-7.

6 Al-Shakarchi NJ, Evans H, Luchenski SA, et al. Cardiovascular disease in homeless versus housed individuals: a systematic review of observational and interventional studies. Heart 2020:106:1483-8.

7 Keulards DCJ, Fournier S, van 't Veer M, et al. Computed tomographic myocardial mass compared with invasive myocardial perfusion measurement. Heart 2020;106:1489-94.

8 Ihdayhid AR, Sellers SL. Novel method for assessing myocardium at risk: a new arrow in the diagnostic quiver of coronary CT. Heart 2020;106:1458-60.

9 Hoole SP, Jenkins DP. Chronic thromboembolic pulmonary hypertension: interventional approaches. Heart 2020;106:1525-31.

10 Kim NH, Delcroix M, Jais X, et al. Chronic thromboembolic pulmonary hypertension. Eur Respir $J$ 2019:53:1801915. 